\title{
Generation and characterization of optical vortices
}

\author{
Luiza R. Vilarta (IC), Luis E. E. Araujo (PQ)
}

\section{Abstract}

We built a spatial light modulator (SLM) by using components from a commercial LCD projector and used the SLM to generate optical vortices. We illuminated a triangular aperture with the optical vortices, and from the observed diffraction pattern, we measured the vortex topological charge.

Key words: Optics, Lasers, Optical Vortices.

\section{Introduction}

Optical vortex beams are light beams that have an azimuthal phase dependance of $\exp (\operatorname{im} \varphi)$, where $m$ is the topological charge of the beam 1 . This kind of beam can be used to rotate and trap particles, create vortex states in Bose-Einstein condensates, among other applications ${ }^{1}$.

The goal of this project was to make optical vortices by projecting a computer-generated holograms $(\mathrm{CGH})^{2}$ onto a home-made SLM ${ }^{2}$. The vortex beams were characterized by observing their diffraction on a triangular apperture ${ }^{3}$.

\section{Results and Discussion}

Our experimental setup is shown in Image 1:

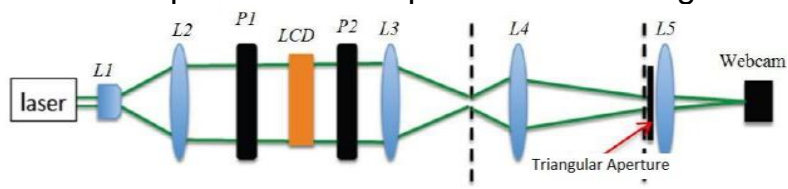

Image 1. Scheme of the experimental setup. L1, L2, L3, L4 and L5 are lenses and P1 and P2 are polarizers.

We modified a commercial multimedia projector, removing one of its LCD screens and placing it on the experimental setup, without disconnecting it from the projector (Image 2). When this screen is between two crossed polarizers, it functions as a SLM.

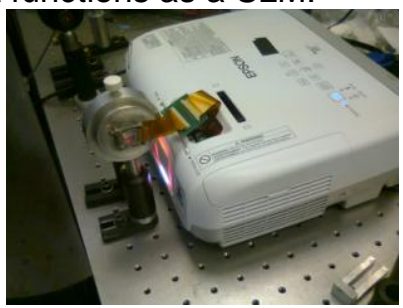

Image 2. Detail of the experimental setup: the LCD screen connected to the Datashow.

By connecting the projector to an auxiliary computer video monitor, the image in the monitor is transfered to the LCD screen. In that way, when a CGH is shown in the auxiliary monitor and the laser passes through the LCD with the hologram, a vortex beam is generated, as shown in Image 3 .
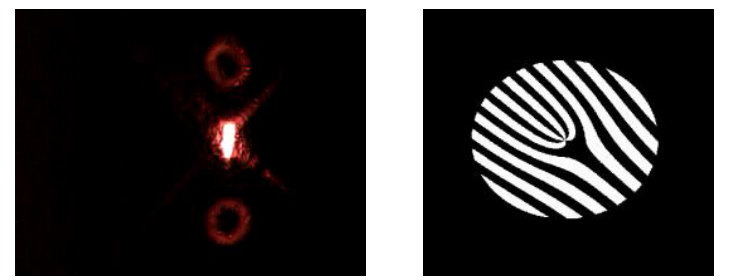

Image 3. Left, a charge $m=4$ optical vortex beam (using a HeNe laser). Right, the $\mathrm{CGH}$ that generated that vortex.

To characterize the vortex beam, we built a triangular apperture and, in the focal plane of lens $L 5$, we put a webcam to visualize the resulting diffraction pattern (Image 4).
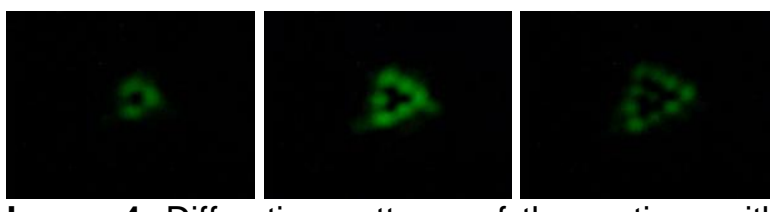

Image 4. Diffraction patterns of the vortices with $m=1$ (left), 2 (center) and 3, (right) after passing through the triangular apperture (using a Nd:YAG laser).

\section{Conclusions}

We have obtained very good results in producing vortex beams with our home-made SLM and were able to characterize the vortex from its diffraction through a triangular aperture.

This experiment can be used in undergraduate laboratories because it is relatively simple to setup and allows real-time observation of other phenomena such as diffraction through various slits.

\section{Acknowledgement}

We thank CNPq and FAPESP for financial support.

\footnotetext{
${ }^{1}$ M. Padgett and L. Allen, Contemp. Phys. 2000, 41, 275.

${ }^{2}$ A. V. Carpentier, H. Michinel, J. R. Salgueiro, and D. Olivieri, Am. J. Phys. 2008, 76, 916.

${ }^{3}$ J. M. Hickmann, E. J. S. Fonseca, W. C. Soares, and S. ChávezCerda, Phys. Rev. Lett. 2010, 105, 053904.
} 